\title{
Hydrodynamical model atmospheres and 3D spectral synthesis
}

\author{
Hans-Günter Ludwig ${ }^{1}$ and Matthias Steffen ${ }^{2}$ \\ 1 CIFIST, GEPI, Observatoire de Paris-Meudon, 92195 Meudon Cedex, France \\ Hans.Ludwig@obspm.fr \\ 2 Astrophysikalisches Institut Potsdam, 14482 Potsdam, Germany \\ msteffen@aip.de
}

\section{Radiation-hydrodynamics modeling - overview}

In this paper we discuss three issues in the context of three-dimensional (3D) hydrodynamical model atmospheres for late-type stars, related to spectral line shifts, radiative transfer in metal-poor 3D models, and the solar oxygen abundance. To establish the context we start by giving a brief overview about the model construction, taking the radiation-hydrodynamics code $\mathrm{CO}^{5} \mathrm{BOLD}$ (COnservative COde for the COmputation of COmpressible COnvection in a $\mathrm{BOx}$ of $\mathrm{L}$ Dimensions with $\mathrm{L}=2,3 ;[3])$ and the related spectral synthesis package Linfor3D as examples.

Based on a Godunov-type finite volume approach, $\mathrm{CO}^{5} \mathrm{BOLD}$ provides the time-dependent solution for a one-component compressible radiating fluid in an external gravity field on a fixed, non-staggered 3D Cartesian grid (allowing variable spacing). Operator splitting separates Eulerian hydrodynamics, optional tensor viscosity, and radiation transport. Directional splitting decomposes the 3D hydrodynamics problem into 1D sub-steps which are treated by an approximate Riemann solver of Roe type, modified to work with an arbitrary equation of state, and to properly handle an external gravity field. This scheme is very robust and well adapted to handle transonic flows and shocks in a highly stratified medium. By design, the code guarantees the numerical conservation of mass, momentum, and energy. For any prescribed chemical composition, $\mathrm{CO}^{5} \mathrm{BOLD}$ uses a tabulated equation of state taking into account partial ionization of $\mathrm{HI}$, He I, and He II, as well as the formation and dissociation of $\mathrm{H}_{2}$ molecules.

The role of radiation in the hydrodynamical simulations is to describe the energy balance due to radiative heating and cooling. The radiative energy exchange is computed from the solution of the non-local transfer equation on a system of a large number of rays traversing the computational volume under different azimuthal and polar angles. Realistic stellar opacities are used, optionally based on ATLAS or MARCS opacity data. The frequency dependence of the radiation field is treated in a multi-group approximation - the so-called opacity binning method (OBM; $[9,7,10])$ - where frequencies are sorted into a small number of bins (typically $4 \ldots 6$ ) according to the ratio 

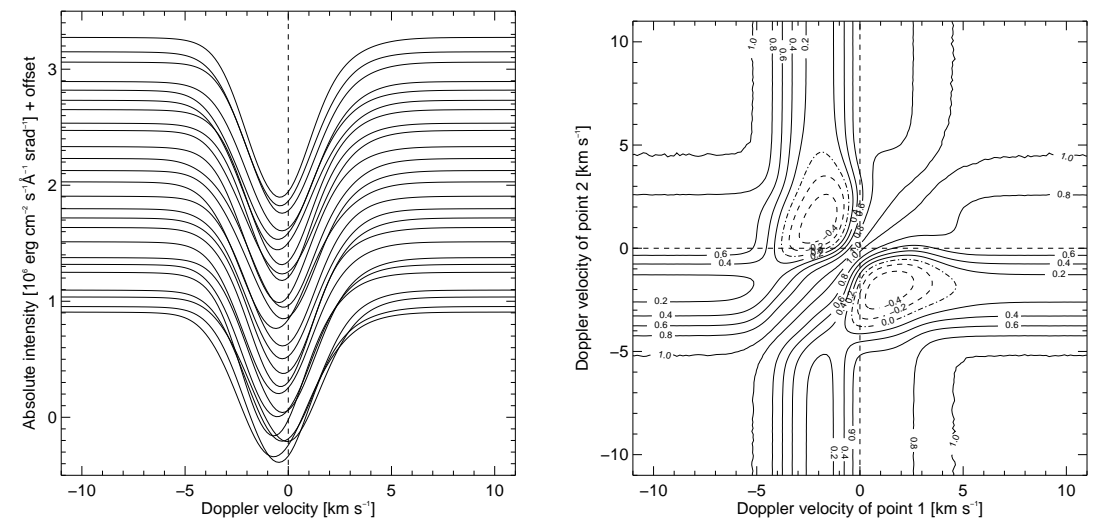

Fig. 1. Left panel: Time series of line profiles of a FeI line at $6082 \AA$ in a 3D solar $\mathrm{CO}^{5} \mathrm{BOLD}$ model. The wavelength is given as corresponding Doppler velocity with respect to the line's laboratory wavelength. The line profiles have been offset proportional to time running from top to bottom. The time interval between profiles is the same, fluctuations in the continuum brightness cause the non-equidistant appearance. Right panel: Contour plot of correlation coefficients of the intensity between two wavelength points in the profiles shown in the left panel.

of monochromatic to Rosseland optical depth. So far, strict LTE is assumed, thus scattering cannot be treated. Radiation pressure is ignored.

The code Linfor3D accepts $\mathrm{CO}^{5} \mathrm{BOLD}$ models as background structures on which spectral synthesis calculations at high wavelength resolution - usually focusing on one particular spectral line - can be performed. When calculating the emergent spectrum, Linfor3D takes into consideration the full 3D flow geometry including Doppler shifts caused by the macroscopic hydrodynamical velocities. It represents the effects of thermal and pressure broadening in standard fashion, but leaves out the ad-hoc broadening mechanisms of micro- and macro-turbulence introduced in 1D atmospheric models. Similar to $\mathrm{CO}^{5} \mathrm{BOLD}$,strict LTE is assumed in Linfor3D. Resulting spectral line profiles provide detailed information about intrinsic line shapes, and convective line shifts with respect to a line's laboratory wavelength.

\section{High precision line shifts from 3D models?}

A $\mathrm{CO}^{5} \mathrm{BOLD}$ simulation constitutes a statistical realization of the atmospheric flow field in the stellar surface layers. If one is not interested in studying time-variable phenomena but only in the mean state of the atmosphere, the fluctuations present a noise source which limits the precision to which flow and related spectroscopic properties can be determined. This is similar to the observational situation where the intrinsic variability of a star limits the precision to which its radial velocity, e.g. in planet searches, can 
be measured. The left panel of Fig. 1 illustrates the temporal variability of a Fe I line calculated from a hydrodynamical solar model. Each line profile is a horizontal average over the surface of the computational box. Shown are 25 instants in time which are sufficiently separated that they can be considered statistically uncorrelated. We ask: what is the precision due to the statistics (ignoring systematic effects) to which we can determine the line shift?

It is straight forward to show (see [8]) that the expectation value of the disk-integrated line profile corresponds to the expectation value of the profile of the local hydrodynamical model. Hence, the statistical uncertainties of the profile emerging from the model directly correspond to the uncertainties of the predicted disk-integrated profile. From Fig. 1 it is obvious that the the statistical fluctuations are not just pixel-to-pixel random noise like in the case of photometric Poisson noise. The line profiles change their overall shape, i.e. different wavelength points show a considerable degree of correlation. The linear correlation coefficient between intensities at two wavelength points 1 and 2 is given by

$$
C\left[I_{1}, I_{2}\right] \equiv \frac{\left\langle\Delta I_{i} \Delta I_{j}\right\rangle}{\sigma_{I_{1}} \sigma_{I_{2}}}=\frac{\left\langle I_{1} I_{2}\right\rangle-\left\langle I_{1}\right\rangle\left\langle I_{2}\right\rangle}{\sigma_{I_{1}} \sigma_{I_{2}}} .
$$

$I$ denotes the intensity, $\langle$.$\rangle the temporal average. \Delta I_{i} \equiv I_{i}-\left\langle I_{i}\right\rangle$ is the intensity deviation from the mean. The right panel of Fig. 1 shows the correlation matrix of the example line depicted.

In order to quantify the line shift $\lambda_{\mathrm{s}}$ we need a model of the procedure by which it is measured, which in turn emerges from the definition of $\lambda_{\mathrm{s}}$. Here, we assume that the measuring procedure of $\lambda_{\mathrm{s}}$ can be described by a function $\Lambda$ of potentially all available (assumed discrete) intensities $I_{i}: \lambda_{\mathrm{s}}=\Lambda\left(I_{i}\right)$. In order to make algebraic headway we simplify and linearize $\Lambda$ around the expectation value of the line profile described by the values $\left\langle I_{i}\right\rangle$. To leading order in $\Delta I$ we obtain for the variance of the line shift the standard expression of the error propagation for correlated variables

$$
\sigma_{\lambda_{\mathrm{s}}}^{2} \approx \sum_{i, j} \frac{\partial \Lambda}{\partial I_{i}} \frac{\partial \Lambda}{\partial I_{j}}\left\langle\Delta I_{i} \Delta I_{j}\right\rangle=\sum_{i, j} \frac{\partial \Lambda}{\partial I_{i}} \frac{\partial \Lambda}{\partial I_{j}} \sigma_{I_{i}} \sigma_{I_{j}} C\left[I_{i}, I_{j}\right] .
$$

The summation is performed over all pixels which are relevant for the measurement of $\lambda_{\mathrm{s}}$. Equation (2) emphasizes the role of the covariance matrix of the intensities $\left\langle\Delta I_{i} \Delta I_{j}\right\rangle$ - or equivalently the standard deviations of the intensities and their correlation matrix - plays for the magnitude of the uncertainty of the line shift. In the present context we discussed line shifts but relation (2) of course also holds for other measures like, e.g., the equivalent width of a line. The statistical quantities in relation (2) can be estimated from the time series provided by the hydrodynamical model. Asymptotically, for a given line one will arrive at a fixed value for the correlation matrix $C\left[I_{i}, I_{j}\right]$. If one wants to improve the accuracy of the line shift one has to 
beat down the uncertainties in the intensities $\sigma_{I_{i}}$. This can be achieved by longer time series or larger horizontal extent of the hydrodynamical model.

Our example FeI line shows a RMS temporal scatter of its position of $0.16 \mathrm{~km} \mathrm{~s}^{-1}$. The value was obtained by directly (and somewhat heuristically) measuring the location of the line core without formalizing the process by explicitely constructing a measurement function $\Lambda$. The statistical independence of the 25 individual snapshots implies an uncertainty of about $30 \mathrm{~m} \mathrm{~s}^{-1}$ for the line shift. While the specific value depends on the chosen line and selection of snapshots we think that it gives an indication of the precision one is typically working with in todays hydrodynamical standard models. Higher precision is possible but computationally also more costly.

Of course, at some point real uncertainties will be dominated by systematic shortcomings of a model.

\section{$33 \mathrm{D}$ radiative transfer in metal-poor atmospheres}

As mentioned earlier, the radiative transfer in the 3D models is commonly approximated by the opacity binning method (OBM) assuming strict LTE. While the approach is working fine in atmospheres of about solar metallicity, metal-poor atmospheres pose a challenge to the OBM. At first glance, this may come as surprise because the dramatic decrease of the number of spectral lines relevant for the radiative energy exchange should simplify the radiative transfer. However, the actual situation is quite different. First, scattering in the continuum becomes important for the thermal structure of metal-poor atmospheres. In the OBM, scattering is treated as true absorption so that one must expect some effects on the resulting temperature structure. Second, experience has shown that the OBM does not work as accurately in metalpoor atmospheres as in atmospheres of solar metallicity. It turned out that this deficit is not related to the treatment of the line blocking but already shows up for the radiative transport in the continuum.

Figure 2 shows an example of an atmosphere of a metal-poor giant. Plotted are temperature profiles of 1D ATLAS6 (see [6]) model atmospheres in radiative-convective equilibrium. The only difference among the models is the way in which the radiative transfer was treated. In three cases labeled "scattering", "no scattering", "scattering as true absorption" a high wavelength resolution was employed, and scattering was treated exactly, scattering opacity was neglected, or treated as true absorption, respectively. The by far dominating scattering opacity under the considered conditions is Rayleigh scattering by hydrogen atoms. As evident from Fig. 2, the temperature structure is noticeably influenced by scattering. The OBM used in the 3D models was also implemented in the $1 \mathrm{D}$ atmosphere code and a resulting radiativeconvective equilibrium calculated. Comparison with the exact radiative transfer solution shows a close correspondence from the deep layers up to lower optical depth of $\log \tau \approx-3$. However, while useful in practice this is only 


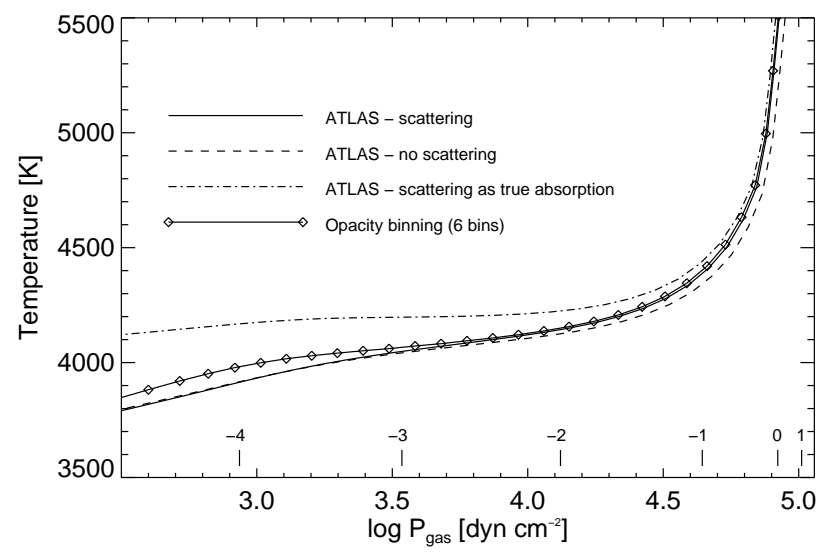

Fig. 2. Comparison of $1 \mathrm{D}$ model atmospheres $\left(T_{\text {eff }}=5000 \mathrm{~K}, \log (g)=2.94\right.$, $[\mathrm{M} / \mathrm{H}]=-2)$ in radiative-convective equilibrium based on different treatments of the radiative transfer. (for details see text)

fortuitous. The OBM based temperature structure should in fact follow the "scattering as true absorption" case since in the OBM scattering opacity is treated as true absorption. At present, the reason for the rather poor performance of the OBM is unclear. Identifying its cause, improving the OBM, and including scattering effects in 3D geometry are challenges to be met in near-future developments of 3D model atmospheres for metal-poor stars.

\section{3D models and the solar oxygen abundance}

Recent spectroscopic abundance determinations by [1], based on a 3D hydrodynamic model atmosphere, led to a much debated downward revision of the solar $\mathrm{C}, \mathrm{N}$, and $\mathrm{O}$ abundances. Their result for the oxygen abundance is $\log \epsilon_{\mathrm{O}}=8.66 \pm 0.05$ (on the scale $\log \epsilon_{\mathrm{H}}=12$ ), causing a dramatic deterioration of the agreement between the thermal structure derived from helioseismic inversions and theoretical solar models, respectively. Motivated by this problem, we (see [2]) are currently using a $3 \mathrm{D} \mathrm{CO}^{5} \mathrm{BOLD}$ simulation with 5-bin frequency-dependent radiative transfer based on MARCS opacities to see whether the results by [1] can be confirmed. This independent redetermination of the solar oxygen abundance is based on 2 forbidden and 7 permitted O I lines, using a number of different observations, including both disk-center ("intensity") and full-disk ("flux") spectra. In addition to 25 snapshots from the simulation, we also derive abundances from different 1D atmospheres for comparison. Special care is taken to provide realistic error estimates.

The following preliminary conclusions can be drawn at this point: (i) "intensity" and "flux" spectra give practically the same result. (ii) the oxygen abundance derived from the $3 \mathrm{D} \mathrm{CO}^{5} \mathrm{BOLD}$ simulation is only slightly lower 
(by -0.04 dex) than that derived from the 1D empirical model by [4] (hereafter $\mathrm{HM}$ ), indicating that the 3D mean model and the 1D HM model have very similar temperature structures in the relevant layers. (iii) the unknown cross sections for neutral particle collisions introduce uncertainties in the NLTE corrections for the O I triplet lines of up to 0.1 dex; depending on the weight of these lines, the resulting error in the mean oxygen abundance derived from our set of lines is about 0.05 dex. (iv) Our preliminary best estimate for the solar oxygen abundance is $\log \epsilon_{\mathrm{O}}=\mathbf{8 . 7 2} \pm \mathbf{0 . 0 6}$, which is close to the value recommended by [5], $\log \epsilon_{\mathrm{O}}=8.736 \pm 0.078$. A remaining problem of our analysis is that the two forbidden [OI] lines give significantly different abundances, which cannot be explained by NLTE-effects or deficiencies of the model atmosphere. We hope to resolve this problem by analyzing the observed center-to-limb variations of these two line profiles.

\section{Remarks on precision spectroscopy and 3D models}

Hydrodynamical model atmospheres are on their way of becoming a standard tool for the analysis of stellar spectra. Their ability of making detailed predictions about the shape of spectral lines in convective atmospheres can only fully exploited if observed spectra of sufficient resolution are available. Ideally, spectrographs should be able to provide a spectral resolutions above $10^{5}$ - something that we would like instrument builders to keep in mind.

High-fidelity abundance work benefits from the theoretical knowledge of the precise intrinsic line shape. However, in practice one is nonetheless often confronted with ambiguities, e.g. in the case of blends, which remain unresolved by considering disk-integrated line profiles only. The center-to-limb variation of a line shape can provide crucial further constraints. Combining interferometry with high-resolution spectroscopy (like in the UVES-I project presented by A. Quirrenbach, this volume) can open-up this source of information for stellar work.

\section{References}

1. M. Asplund, N. Grevesse, A.J. , Sauval, C. Allende Prieto, D. Kiselman, A\&A 417, 751 (2004)

2. E. Caffau, H.-G. Ludwig, M. Steffen, T.R. Ayres, R. Cayrel, P. Bonifacio, B. Plez, B. Freytag: A\&A, (2007), in preparation

3. B. Freytag, M. Steffen, B. Dorch, AN 323, 213 (2002)

4. H. Holweger, E. Müller, Solar Phys. 39, 19 (1974)

5. H. Holweger, in: Solar and galactic composition, ed by R.F. WimmerSchweingruber, AIP Conf. Proc. 598, p. 23 (2001)

6. R.F. Kurucz, ApJ 40, 1 (1979)

7. H.-G. Ludwig, S. Jordan, M. Steffen. A\&A 284, 105 (1994)

8. H.-G. Ludwig, A\&A 445, 661 (2006)

9. A. Nordlund, A\&A 107, 1 (1982)

10. A. Vögler, J.HM.J. Bruls, M. Schüssler, A\&A 421, 741 (2004) 